\title{
Procedimiento y Evaluación del Prácticum en la Facultad de Educación de la Universidad de León
}

\section{Procedure and Evaluation of Practicum in the Faculty of Education in the University of León}

\author{
Dra. Ana María de Caso Fuertes \\ Vicedecana de Prácticum de la Facultad de Educación \\ Universidad de León \\ fcevdec2@unileon.es
}

\section{María Dolores Alonso-Cortés Fradejas}

Vicerrectora de Relaciones Institucionales y con la Sociedad

Universidad de León

mdalof@unileo.es

Caso Fuertes, A., Alonso-Cortés Fradejas, M.D. (2017). Procedimiento y Evaluación del Prácticum en la Facultad de Educación de la Universidad de León. Revista Prácticum, Vol1 (1) 114-122. 2530-4550 


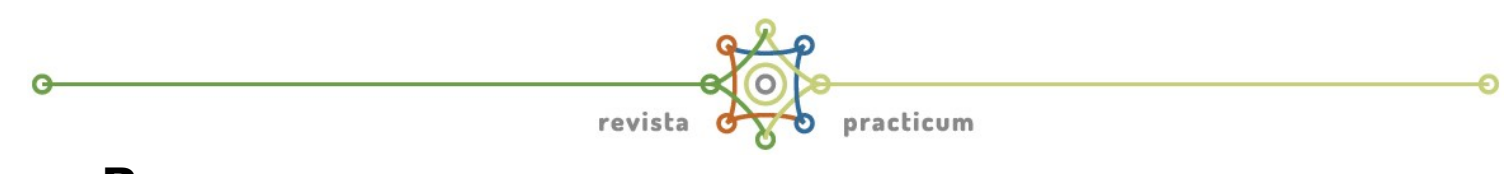

\section{Resumen}

El presente trabajo tiene como objetivo dar a conocer no sólo la evaluación de las asignaturas Prácticum de la Facultad de Educación de la Universidad de León, sino también cuál es su implementación y procedimiento. De este modo se deja constancia de las numerosas personas que forman parte del proceso, lo que provoca la necesidad de una evaluación compartida y cooperativa que exige un incremento de transparencia, imparcialidad y aceptabilidad a fin de optimizar el proceso de enseñanza del alumno, de modo que él mismo sea el protagonista de su aprendizaje. Para ello se vienen mejorando dos tipos de rúbricas diferentes: una que evidencia las competencias necesarias para la realización de la memoria o informe final, y otra que facilita la valoración de la estancia en los centros del estudiante por parte de su maestro-tutor y coordinador. Del mismo modo, a pesar de utilizar diferentes canales de comunicación interactiva con los alumnos (moodle) y entre profesores (otras plataformas interactivas de la Consejería de Educación de la Junta de Castilla y León), se hace patente la necesidad de transformar esas rúbricas en erúbricas que fomenten la retroalimentación de los aprendizajes.

\section{Abstract}

This paper aims to present not only the evaluation of the subjects Practicum of the Faculty of Education of the University of León, but also what is its implementation and procedure. In this way, the many people who form part of the process are recorded, which provokes the need for a shared and cooperative evaluation that requires an increase of transparency, impartiality and acceptability in order to optimize the student's teaching process That he himself is the protagonist of his learning. Two different types of rubrics have been improved: one that demonstrates the skills required for the completion of the report or final report, and another that facilitates the evaluation of the stay in the student centers by their teachertutor and coordinator. Similarly, despite the use of different channels of interactive communication with students (moodle) and between teachers (other interactive platforms of the Council of Education of Castilla y León), the need to transform these rubrics in Erubrics that encourage the feedback of learning.

\section{Palabras claves}

Practicum, Facultad de Educación, Organización Escolar, Evaluación, Evaluación aprendizajes, Rúbricas. 


\section{Keywords}

Practicum, Education Faculty, School Organization, Evaluation, Assessment, Rubrics.

\section{Introducción: marco contextual}

El proceso de convergencia europea supuso una transformación en las instituciones universitarias, quienes han desempeñado un importante papel en la construcción del Espacio Europeo de Educación Superior (Pozo \& García, 2006). Dicho proceso se basaba en la idea de que sólo se logra un aprendizaje eficaz siendo el alumno el responsable de la organización y desarrollo de su trabajo académico, por lo que debe promoverse el autoaprendizaje del estudiante, resaltando su protagonismo, tanto en la adquisición de contenidos instrumentales y teóricos como en una serie de competencias transversales reconocidas como esenciales (Blanco, 2009).

A este fin, la Facultad de Educación de la Universidad de León (ULE en adelante), en sesión de Junta de Facultad celebrada el 20 de noviembre de 2007, crea una Comisión encargada de elaborar los Planes de Estudio de los Grados en Educación Primaria y en Educación Infantil, resultando en la redacción de ambas Memorias de Verificación, informadas favorablemente por la ANECA el 25 de junio de 2009, publicándose sendos planes de estudios en el BOE del 27 de mayo de 2011. Ambas Memorias de Verificación se ajustan a las disposiciones establecidas por el Gobierno al haber sido atendidos los requisitos establecidos en la ORDENECI/3854/2007 y en el Anexo correspondiente (BOE 29 de diciembre de 2007): denominación, objetivos, planificación de las enseñanzas, módulo de formación básica, módulo didáctico y disciplinar y Prácticum, con la asignación crediticia establecida en cada caso.

En concreto, los objetivos propuestos, y aprobados, para las asignaturas de prácticum de los grados de Educación Infantil y Educación Primaria fueron (1) Adquirir un conocimiento práctico del aula y de la gestión de la misma; (2) Conocer y aplicar los procesos de interacción y comunicación en el aula, así como dominar las destrezas y habilidades sociales necesarias para fomentar un clima que facilite el aprendizaje y la convivencia; (3) Controlar y hacer el seguimiento del proceso educativo $y$, en particular, de enseñanza y aprendizaje mediante el dominio de técnicas y estrategias necesarias; (4) Relacionar teoría y práctica con la realidad del aula y del centro; (5) Participar en la actividad docente y aprender a saber hacer, actuando y reflexionando desde la práctica; (6) Participar en las propuestas de mejora en los distintos ámbitos de actuación que se puedan establecer en un centro; (7) Regular los procesos de 
revista $\frac{7}{8}$ practicum

interacción y comunicación en grupos de alumnos de 0-3 años y de 36 años, en el caso del Grado de Educación Infantil y de 6 a 12 años en el caso del Grado de Educación Primaria; y un último objetivo en éste último Título mencionado, que hace referencia a (8) Conocer formas de colaboración con los distintos sectores de la comunidad educativa y del entorno social.

\subsection{Normativa legal}

Las Órdenes Ministeriales ECI/3854/2007 y ECI/3857/2007, ambas del 27 de diciembre, ya conceden especial relevancia al desarrollo de las asignaturas del prácticum en centros de Educación Infantil o Primaria, por lo que el Ministerio de la Presidencia dispone el Real Decreto 1707/2011, de 18 de noviembre, ratificado por el Real Decreto 592/2014, de 11 de julio, que regulan las prácticas académicas externas de los estudiantes universitarios.

A raíz de estas órdenes, la Consejería de Educación de la Junta de Castilla y León, a través de la Orden EDU/9/2012 de 13 de enero, regula con carácter experimental la realización de las prácticas de las enseñanzas universitarias de grado en Educación Infantil y de Grado en Educación Primaria, y que desemboca en la normativa actual regulada por la Orden EDU/641/2012 de 25 de Julio, que ya establece la forma de realizar las prácticas de las asignaturas del prácticum de los Grados mencionados anteriormente en los centros docentes sostenidos con fondos públicos de Castilla y León.

Esta última Órden EDU/641/2012 determina los requisitos y funciones que deben cumplir todos los participantes del prácticum (Figura 1), además de establecer dos Comisiones que coordinen y gestionen el mismo:

(1) Comisión Provincial de Prácticas de Grado, responsable de la coordinación insterinstitucional y de gestión de la participación de los sectores universitario y escolar en el desarrollo del prácticum en los centros escolares de cada provincia.

(2) Comisión Regional de Prácticas de Grado, encargada de la coordinación interinstitucional, de deliberación, decisión y gestión de la participación de los sectores universitario y no universitario en el desarrollo estratégico del prácticum a nivel regional, y adscrita, por tanto, a la Consejería de Educación. 


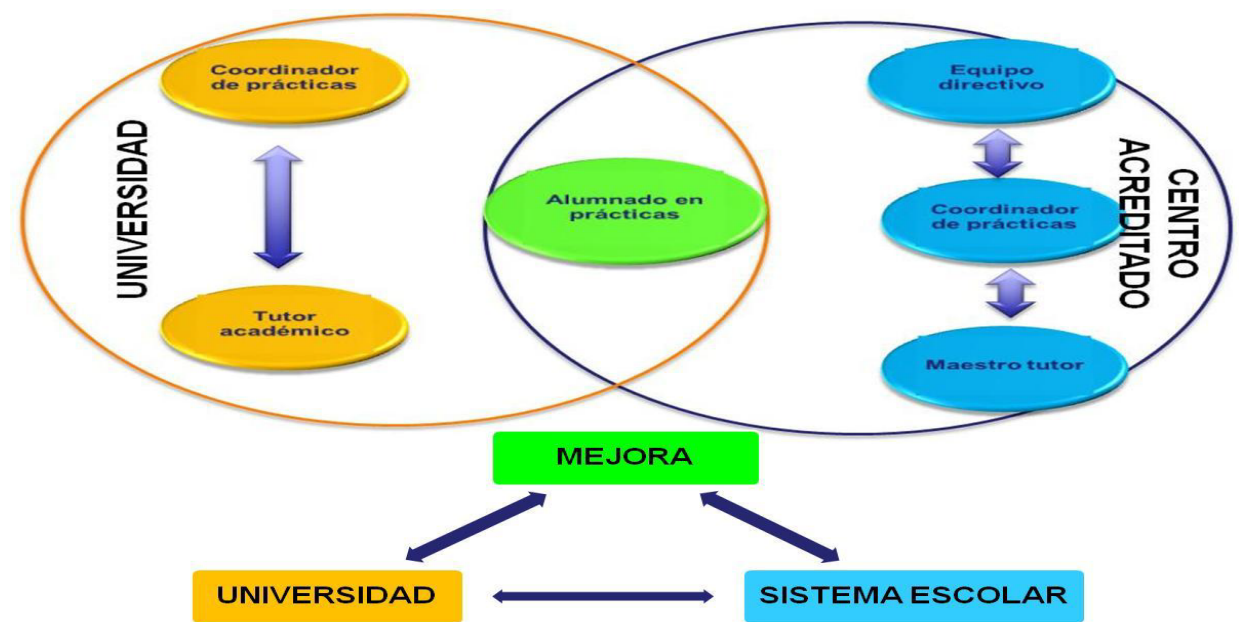

Figura 1. Participantes en el prácticum según la Orden EDU/641/2012, de 25 de julio

Desde el marco de esta normativa legal, la ULE acuerda en Consejo de Gobierno del 16/7/2012 (modificado en Consejos de Gobierno del 8/11/2012, 17/6/2014 y 2/10/2014), aprobó el Reglamento por el que se regulan las prácticas externas en los estudios de Grado, Máster y Doctorado de la Universidad de León.

\section{Procedimiento de prácticum}

Los planes de estudio de ambos grados de Educación (Infantil y Primaria) incluyen dos asignaturas prácticas: el Prácticum I, de 20 créditos, que se realizaría en el primer cuatrimestre del tercer curso, y el Prácticum II, de 22 créditos, que se llevaría a cabo en el segundo cuatrimestre del último curso. Destacar que todos ellos tienen un carácter generalista, a excepción del Prácticum II del Grado de Educación Primaria que está vinculado a una mención (lengua inglesa, educación física, educación musical, educación especial, o audición y lenguaje).

Estos prácticums se desarrollan en tres fases, una primera fase preparatoria cuyo objetivo es planificar y preparar la actividad que desarrollarán los alumnos durante su estancia en los centros; una segunda fase de prácticas en los centros educativos, donde los alumnos deben permanecer en las aulas durante 8-9 semanas aproximadamente bajo la tutoría de un maestro y la supervisión del coordinador del prácticum en el centro escolar correspondiente; y una última fase de evaluación donde participan tanto los coordinadores de prácticas de los centros educativos como los profesores de la ULE que imparten docencia en estas asignaturas, como la Vicedecana de Prácticum.

La fase preparatoria pretende preparar alumnado tanto para el desarrollo autónomo de las competencias relacionadas con la materia Prácticum, como para el aprendizaje mediante la observación, a la 
vez que se le inicia en la práctica docente (pautas de intervención, discurso de aula, etc.) y se le familiariza con los distintos contextos en los que se van a realizar prácticas (tipos de centro, programas institucionales, organización escolar, etc.). Para ello se realizan dos tipos de actividades formativas: Conferencias durante las que docentes invitados de varios centros de León hablarán al alumnado de distintos aspectos de la realidad educativa (atención a la diversidad, bilingüismo, la organización de un centro educativo, el trato con las familias, la resolución de conflictos...), y Sesiones generales en las que el profesorado de la ULE proporciona al alumnado una serie de pautas generales para el análisis de los procesos educativos y para la transferencia a un informe final de los aprendizajes realizados durante la estancia en los centros.

Por otro lado, mientras el estudiante realiza las prácticas observando, reflexionando e interviniendo en los centros educativos, se realiza un seminario semanal en la Facultad de Educación donde los alumnos comparten las experiencias vividas y aprendizajes realizados a lo largo de la semana en el centro correspondiente, de modo que pueden analizar posibles diferencias de la realidad educativa según el contexto del centro escolar. Esta sesión semanal, al igual que el resto de actividades, es obligatoria para el estudiante, de modo que estarían exentos de asistir esa tarde al centro escolar e (si éste impartiese clase por la tarde). Además, durante esta segunda fase, se realiza un seguimiento del alumno por parte del profesorado de la ULE, mediante entrevistas al tutor del centro correspondiente, cuestionarios al propio alumno, supervisión del portafolios o la memoria, y al menos una visita al centro escolar.

Cabe decir, que tanto los centros educativos a los que acuden los alumnos matriculados en cualquier prácticum, como los tutores que se les asignen en los respectivos centros, han de estar debidamente acreditados como centros de formación en prácticas y maestro tutor de prácticas respectivamente, por la Consejería de Educación de la Junta de Castilla y León; por lo que la asignación de los alumnos a los diferentes centros se realiza mediante un laborioso proceso en el que participan tanto la Vicedecana de Prácticum de la Facultad de Educación de la ULE como el Técnico correspondiente de la Dirección Provincial de León, que es quien proporciona los centros y maestros acreditados.

\section{Evaluación del prácticum}

A lo largo de estos años de implantación de los Grados de Educación Infantil y Primaria, se ha ido intentando mejorar el sistema de evaluación del Prácticum en aras de una mayor objetividad y transparencia en la calificación final del alumno, ya que son varios los agentes que participan de esa evaluación. 
Para superar cualquiera de estas asignaturas el alumno ha de aprobar tres tipos de actividades formativas: las conferencias y sesiones de preparación (que suponen el $10 \%$ de la calificación final), las tareas de análisis en las sesiones simultáneas y el informe final, valorado por los profesores del Prácticum de la ULE (implicando un $45 \%$ de la nota final), y las prácticas en los centros evaluadas tanto por el maestro-tutor como por el coordinador del centro correspondiente (que son el otro $45 \%$ restante).

Puesto que son varios los profesores de la ULE que supervisan a alumnos de prácticum, y números los maestros tutores que califican a los alumnos en los centros (cada maestro-tutor tiene un único alumno en prácticas, lo que hace un total de unos 350 tutores aproximadamente), se hace imprescindible el uso de una rúbrica que unifique criterios y aporte imparcialidad, transparencia y aceptabilidad a las calificaciones finales del prácticum (Baartman, Bastiaens. Kirschener y Van der Vleuten, 2006; Gallego y RaposoRivas, 2014).

Al igual que Fernández (2010), entendemos la rúbrica como una "guía de puntuación usada en la evaluación del estudiante, que describe las características específicas de un trabajo en varios niveles de rendimiento", de modo que se clarifica lo que se espera del alumno, a la vez que se objetiviza la evaluación realizada por agentes diferentes al incluir indicadores y evidencias ponderadas a los que se asignan los criterios (Cebrián y Monedero, 2014). No en vano, como prueban Valverde y Ciudad (2014) "puntuar con una rúbrica es probablemente más fiable que puntuar sin ella", al permitir a los docentes y estudiantes conocer y compartir los indicadores, criterios y evidencias.

Concretamente, en la ULE, se han desarrollado, y se siguen optimizando curso a curso, dos tipos de "rúbricas" diferentes: (1) una que auna y clarifica los criterios de calificación de los informes de los estudiantes, de modo que se les "exija" a todos los mismo en términos no sólo de contenidos sino de competencias de cada uno de los prácticums, independientemente de quién sea el profesor que califique la memoria; y (2) otra que auna y clarifica los criterios de evaluación y competencias que deben valorar los maestros-tutores de los centros, los cuales, según el RD 592/2014 de 11 julio, deben remitir un informe al tutor académico donde valoren tanto las competencias genéricas como las específicas y otros aspectos como la capacidad técnica, la capacidad de aprendizaje, el sentido de la responsabilidad, la facilidad de adaptación, la creatividad e iniciativa...etc. Ambos documentos de evaluación se encuentran a disposición tanto de alumnos como de tutores académicos como de maestros-tutores a través de la plataforma interactiva moodle, pero no se puede considerar una "e-rúbrica" dado que no existe comunicación ni retroalimentación en este sentido entre alumnos y 
profesores (Cebrián, Serrano, y Ruíz, 2014) aspecto éste objeto de mejora.

La primera de las "rúbricas", relacionada con la evaluación del profesorado académico, muy susceptible de mejora en cuanto a clarificación de indicadores, difiere según la asignatura de la que estemos hablando (Prácticum I o II, de Infantil o Primaria), de modo que contiene, a parte de los requisitos formales establecidos para cada caso con su correspondiente valoración, distintos apartados referidos bien a "descripción y características generales del centro" donde se realizan las prácticas, bien a "análisis de los procesos de enseñanza-aprendizaje", bien a "justificación de un proyecto de innovación" y su posterior "intervención o puesta en marcha", finalizando todas ellas con una "valoración personal del alumno".

La segunda de las rúbricas, dirigida a la evaluación de las competencias del alumno por parte del maestro-tutor y el coordinador del centro, ha ido mejorando considerablemente a lo largo de los cursos, desembocando en un documento que evalúa aspectos como "la adquisición de un conocimiento práctico del aula y de la gestión de la misma", "el conocimiento y aplicación de los procesos de interacción y comunicación en el aula, y dominio de las destrezas y habilidades sociales necesarias para fomentar un clima de aula que facilite el aprendizaje y la convivencia", "el control y seguimiento del proceso educativo y en particular el de enseñanzaaprendizaje mediante el dominio de las técnicas y estrategias necesarias", o la "regulación de los procesos de interacción y comunicación" (aspectos éstos que evalúa el maestro-tutor), y otros indicadores más formales como puntualidad, actitud de colaboración con el centro y respuesta a las indicaciones, que evalúa el coordinador.

\section{Referencias bibliográficas}

Baartman,L.K.J., Bastiaens, T.J., . Kirschener, P.A., \& Van der Vleuten, C.P.M. (2006). The Wheel of competency assessment: Presenting quality criteria for competency assessment programs. Studies in Educational Evaluation, 32 (2), 153-170.

Blanco, A. (2009). Desarrollo y evaluación de competencias en educación superior. Madrid: Narcea.

Cebrián, M. \& Monedero, J. J. (2014). Evolución en el diseño y funcionalidad de las rúbricas: desde las rúbricas "cuadradas" a las erúbricas federadas. REDU: Revista de Docencia Universitaria, 12 (1), 81-98.

Cebrián, M., Serrano, R. J. \& Ruíz, M. (2014). Las eRúbricas en la evaluación cooperativa del aprendizaje en la Universidad. Comunicar, 43, 153-161. 
Fernández, A (2010). La evaluación orientada al aprendizaje en un modelo de formación por competencias en la educación universitaria. REDU: Revista de Docencia Universitaria, 8 (1), 11-34.

Gallego, M. J. \& Raposo-Rivas, M. (2014). Compromiso del estudiante y percepción del proceso evaluador basado en rúbricas. REDU: Revista de Docencia Universitaria, 12 (1), 197-215.

Pozo. M. T., \& García, B. (2006). El portafolios del alumnado: una investigación-acción en el aula universitaria. Revista de Educación, 341, 737-756.

Valverde, J. \& Ciudad, A. (2014). El uso de e-rúbricas para la evaluación de competencias en estudiantes universitarios. Estudio sobre la fiabilidad del instrumento. REDU: Revista de Docencia Universitaria, 12 (1), 49-79. 